\title{
Chloronychia: green nail syndrome caused by Pseudomonas aeruginosa in elderly persons
}

\author{
This article was published in the following Dove Press journal: \\ Clinical Interventions in Aging \\ 14 January 2015 \\ Number of times this article has been viewed
}

\section{Anca Chiriac ${ }^{1-3}$ \\ Piotr Brzezinski ${ }^{4}$ \\ Liliana Foia ${ }^{5}$ \\ losif Marincu ${ }^{6}$}

'Apollonia University, ${ }^{2}$ Nicolina Medical Center, Department of Dermatology, 3"P.Poni” Institute of Macromolecular Chemistry, lasi, Romania; ${ }^{4}$ Department of Dermatology, 6th Military Support Unit, Ustka, Poland; ${ }^{5}$ Grigore T. Popa University of Medicine and Pharmacy, lasi, Romania; ${ }^{6}$ Victor Babes University of Medicine and Pharmacy, Timisoara, Romania
Correspondence: Anca Chiriac Apollonia University, Nicolina Medical Center, Department of Dermatology, P Poni Institute of Macromolecular Chemistry, Strada Muzicii nr 2, lasi, Romania

Tel +407 2I234999

Email ancachiriac@yahoo.com

\begin{abstract}
Green nails, also known as chloronychia or green nail syndrome, are characterized by green discoloration of the nail plate (greenish-yellow, greenish-brown, greenish-black), proximal chronic non-tender paronychia, and distolateral onycholysis. The cause is Pseudomonas aeruginosa infection of the nail plate in persons whose hands are constantly exposed to water, soaps, and detergents or are subject to mechanical trauma, especially in the elderly. Green or black coloration of the nails should raise suspicion for Pseudomonas infection and be treated with an oral quinolone (ciprofloxacin), particularly in aged patients. We present three cases of green nails in elderly persons.
\end{abstract}

Keywords: chloronychia, green nail syndrome, Pseudomonas aeruginosa, elderly

\section{Case series Case I}

A 67-year-old healthy retired man developed an asymptomatic greenish discoloration of the nail plate of the right toe nail over a period of 2 months. There was a history of chronic nail trauma prior to the discoloration, probably due to tight shoes. Dermatologic examination showed greenish-black discoloration, mild onychodystrophy of the entire nail plate, and distal onycholysis (Figure 1).

Bacteriologic culture of nail scrapings was positive for Pseudomonas aeruginosa. Fungal coinfection was excluded by potassium hydroxide preparation and culture. The nail plate was cured after 6 weeks of oral ciprofloxacin.

\section{Case 2}

A 68-year-old gardener was seen during summer, after working in wet conditions, for green discoloration of his right finger nail, slight pruritus, and pain. On clinical examination, distal onycholysis and greenish discoloration were noticed on the nail plate starting from the distal edge (Figure 2).

Bacteriologic culture of nail scrapings was positive for P. aeruginosa. No fungal coinfection was proved. Cutting the distal part of the nail plate and application of topical gentamicin twice daily for 1 month were followed by positive results.

\section{Case 3}

A 56-year-old man, diagnosed 1 year earlier with toenail onychomycosis, in the absence of any treatment, developed greenish discoloration of the entire nail plate. No signs of paronychia were noticed, only subungual hyperkeratosis (Figure 3).

Bacteriologic culture of nail scrapings was positive for $P$. aeruginosa. Direct mycologic examination of nail scrapings was positive and culture was positive for 


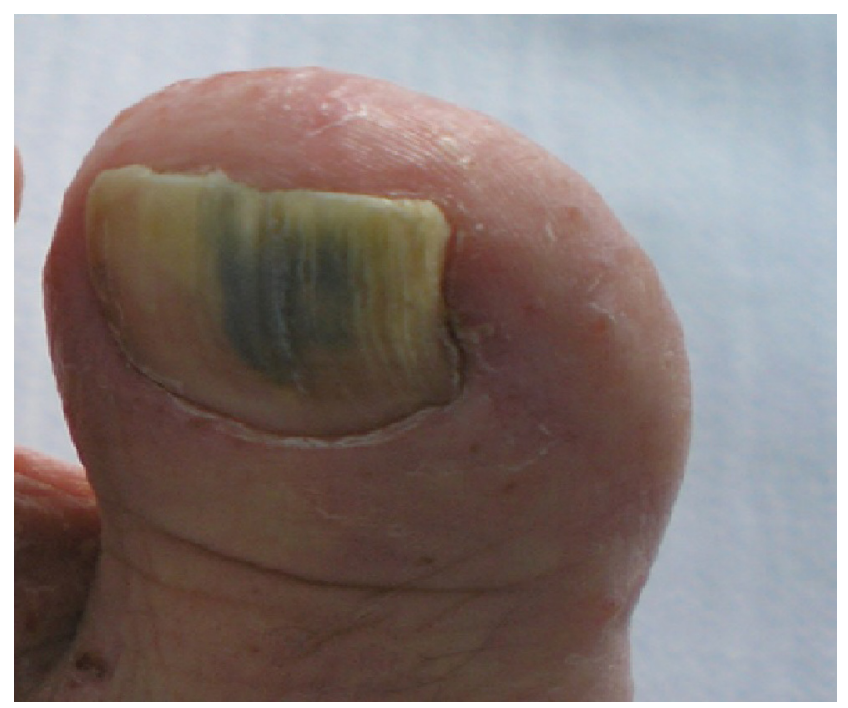

Figure I Green coloration of the toe nail.

Trichophyton rubrum. The fungal infection was treated with itraconazole $200 \mathrm{mg} /$ day 14 days per month for 3 months. Bacterial infection was cured after systemic administration of ciprofloxacin $500 \mathrm{mg} /$ day for 3 weeks.

\section{Discussion}

Bacterial infections of the nails are caused by Gramnegative bacteria, usually $P$. aeruginosa, but can also be caused by Klebsiella spp. and Gram-positive bacteria like Staphylococcus aureus. ${ }^{1,2}$ P. aeruginosa is the most common pathogen causing bacterial nail infections, although it is rarely reported. ${ }^{3} P$. aeruginosa is a Gram-negative, aerobic, coccobacillus belonging to the Pseudomonadaceae family.

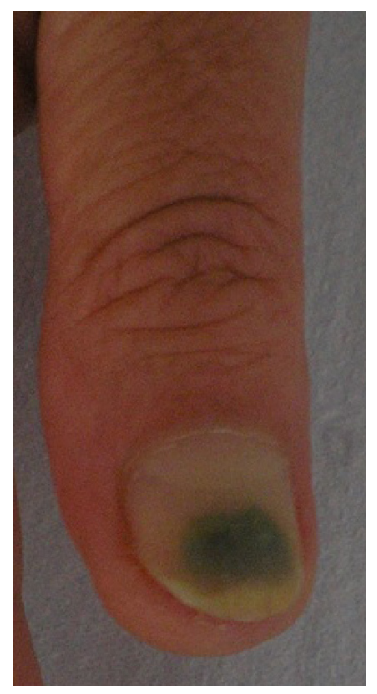

Figure 2 Onycholysis and secondary Pseudomonas aeruginosa infection of the finger nail in a 68-year-old man.

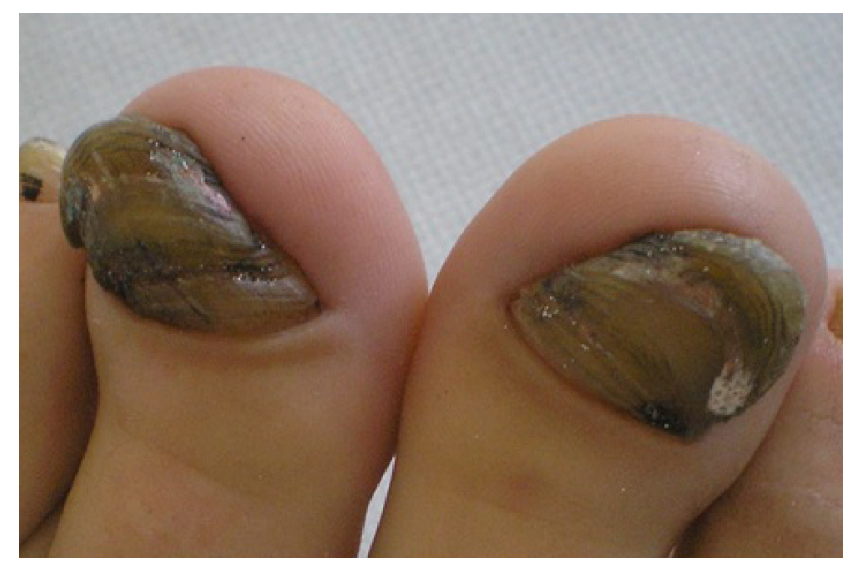

Figure 3 Onychomycosis, onychogryphosis, and choloronychia in a 56-year-old man. Note: Colonies of Pseudomonas aeruginosa grown on agar media.

These pathogens are widespread in nature, inhabiting soil, water, plants, and animals (including humans).

More than half of all clinical isolates produce the bluegreen pigments pyoverdin and pyocyanin. ${ }^{2} P$. aeruginosa is an opportunistic human pathogen that can produce pulmonary, kidney, and urinary tract infections and even systemic infections. It can be also involved in soft tissue infections, skin and nail infections in immunocompetent subjects and patients with immune deficiency syndrome. $P$. aeruginosa is not part of normal skin flora, so Pseudomonas infections of the intact nail are rare. When infection occurs, $P$. aeruginosa colonizes moist regions of the skin, axillae, anogenital regions, and retroauricular areas.

The pathogenesis of nail infections caused by $P$. aeruginosa is not fully elucidated. Predisposing factors must be taken into consideration: onycholysis, onychotillomania, microtrauma to the nail-fold, chronic paronychia, chronic exposure to water, soaps, or detergents, and associated nail disorders, such as psoriasis. ${ }^{4}$

Onycholysis is characterized by separation of the nail plate from the nail bed; it is followed by secondary Pseudomonas infection, particularly when the nails are exposed to a warm and moist environment. A number of triggers for onycholysis have been reported, including psoriasis, onychomycosis, yellow nail syndrome, contact dermatitis, medications (doxycycline), endocrine disorders (acquired hypoparathyroidism), ${ }^{5}$ constant local trauma, especially in elderly. Chloronychia is more common in homemakers, barbers, dishwashers, bakers, and medical personnel, and can be regarded as an occupationally triggered disease. ${ }^{6}$

Small cuttings from the affected nail and/or subungual debris should be sent to the laboratory for investigations. Gram stain coloration is performed presenting Gram-negative rods 
with no particular arrangement; on cetrimide agar medium, $P$. aeruginosa expresses pyocyanin, a blue-green exopigment, and the colonies are flat, large, and oval, with a characteristic fruity smell.

Recently it has been reported a strong relation between fungal and $P$ Aeruginosa infection of the nail: fungal infection stimulates bacterial colonization within the nail and overgrowth of $P$ Aeruginosa in culture inhibits the isolation of fungus. ${ }^{7}$ The differential diagnosis includes subungal hematoma, malignant melanoma, infections caused by other pathogens such as Aspergillus, Candida, and Proteus, and chemical exposure to solutions containing pyocyanin or pyoverdine. ${ }^{8}$

The treatment of chloronychia, especially in elderly people, is difficult in many cases and recommendations based on clinical trials are missing. ${ }^{3}$ In the past, removal of the entire nail was a therapeutic option, but not nowadays. Treatment consists of cutting off the detached nail plate, brushing the nail bed with a $2 \%$ sodium hypochlorite solution twice daily, prevention of repeated immersion by wearing cotton and latex gloves and antibiotics administered topically and orally.

Topical silver sulfadiazine, ciprofloxacin, and gentamicin have also been reported to be valuable therapeutic options. ${ }^{9}$ Topical antibiotics (polymyxin B or bacitracin) applied 2-4 times daily for 1-4 months have been demonstrated to be effective in immunocompetent patients. ${ }^{6}$ Topical therapy with nadifloxacin applied once daily for several weeks was curative for two patients with acquired immune deficiency syndrome. ${ }^{4}$ When topical therapies are not preferred by the patient, oral ciprofloxacin should be recommended for 2-3 weeks.

Pseudomonas infections of the nails are treated by fluoroquinolones, when necessary, in comparison with other bacterial nail infections that require culture and sensitivity testing. This is the reason to recognize easily, by simple clinical observation of the color of the nails, especially in aged persons, to avoid unnecessary laboratory investigations and to save time.

\section{Conclusion}

Green coloration of the nails should raise suspicion for Pseudomonas infection. Treatment with an oral quinolone (ciprofloxacin), particularly in aged patients when laboratory investigations are difficult to perform, could be a valuable option.

\section{Disclosure}

The authors report no conflicts of interest in this work.

\section{References}

1. Maes M, Richert B, de la Brassinne M. Green nail syndrome or chloronychia. Rev Med Liege. 2002;57(4):233-235.

2. Pier GB, Ramphal R. Pseudomonas aeruginosa. In: Mandell GL, Bennett JE, Dolin R, editors. Principles and Practice of Infectious Diseases. 6th ed. Philadelphia, PA, USA: Churchill Livingstone; 2004.

3. Nenoff P, Paasch U, Handrick W. [Infections of finger and toe nails due to fungi and bacteria]. Hautarzt. 2014;65(4):337-348.

4. Rallis E, Paparizos V, Flemetakis A, Katsambas A. Pseudomonas fingernail infection successfully treated with topical nadifloxacin in HIVpositive patients: report of two cases. AIDS. 2010;24(7):1087-1088.

5. Sarkar S, Mondal M, Das K, Shrimal A. Mucocutaneous manifestations of acquired hypoparathyroidism: an observational study. Indian $J$ Endocrinol Metab. 2012;16(5):819-820.

6. Greene SL, Su WP, Muller SA. Pseudomonas aeruginosa infections of the skin. Am Fam Physician. 1984;29(1):193-200.

7. Yang YS, Ahn JJ, Shin MK, Lee MH. Fusarium solani onychomycosis of the thumbnail coinfected with Pseudomonas aeruginosa: report of two cases. Mycoses. 2011;54(2):168-171.

8. Cheung F. A strange looking toenail. Journal of Current Clinical Care. 2013;3(6):22-24.

9. Agger WA, Mardan A. Pseudomonas aeruginosa infections of intact skin. Clin Infect Dis. 1995;20(2):302-308.
Clinical Interventions in Aging

\section{Publish your work in this journal}

Clinical Interventions in Aging is an international, peer-reviewed journal focusing on evidence-based reports on the value or lack thereof of treatments intended to prevent or delay the onset of maladaptive correlates of aging in human beings. This journal is indexed on PubMed Central, MedLine,

\section{Dovepress}

CAS, Scopus and the Elsevier Bibliographic databases. The manuscript management system is completely online and includes a very quick and fair peer-review system, which is all easy to use. Visit http://www.dovepress. com/testimonials.php to read real quotes from published authors. 